\title{
Superplasticity in a magnesium alloy prepared with bimodal grain size distributions developed by dynamic recrystallisation
}

\author{
J. A. del Valle and O. A. Ruano \\ Dept. of Physical Metallurgy, Centro Nacional de Investigaciones Metalúrgicas (CENIM), \\ CSIC, Avda. de Gregorio del Amo 8, 28040 Madrid, Spain
}

\begin{abstract}
In the present work the influence of bimodal grain size distributions on superplastic behavior, of a magnesium alloy, was investigated. Samples with different volume fraction of fine grains have been prepared, and their strain rate-stress relation during superplasticity has been measured. Additionally, the predictions of two deformation models, based on the isostrain and the isostress conditions, were compared with the experimental data. The isostrain model allows the major experimental observations to be readily explained and predicted.
\end{abstract}

Keywords: Microstructure; Mechanical properties; Magnesium alloys; Grain boundary sliding; Superplasticity; Bimodal grain size distributions

\section{Introduction}

Superplastic forming is an interesting procedure of shaping Mg alloys. Thus, significant efforts are being lately devoted to understand the underlying physical mechanisms that take place during superplastic deformation of these materials. Moreover, several investigations are focused on optimizing grain refinement processing routes using severe plastic deformation techniques. As a final result of these processing routes, a bimodal grain size microstructure has been often obtained [1, 2]. The influence of such microstructures on superplasticity is currently 
under discussion $[3,4]$.

In the present work the influence of bimodal grain size distributions on superplastic behavior, of the magnesium alloy AZ31, was investigated. Samples with bimodal grain size distributions have been prepared taking advantage of dynamic recrystallisation induced by application of a tensile pre-deformation [5]. Successively, the strain rate-stress relation has been investigated by means of strain rate change tests. Additionally, two theoretical models have been considered namely: an isostrain model [6] and an isostress model [7]. Both models have been used in the literature for describing creep of such bimodal microstructures and the transition from slip creep to grain boundary sliding (GBS) [6, 7, 8].

\section{Experimental}

The material used in this study was a commercial Mg alloy AZ31 with the following composition: 3\%Al, 1\%Zn, $0.2 \% \mathrm{Mn}$ and $\mathrm{Mg}$ (balance), provided by Magnesium Elektron in the form of a sheet, $3 \mathrm{~mm}$ in thickness. The alloy was received in the condition AZ31-O (rolled and annealed). Flat tensile samples of $10 \mathrm{~mm}$ gage length were cut out from the as-received material. Samples A to D were produced by successive deformation steps, consisting in the application of a tensile pre-deformation and re-machining of the gauge length, as given in Table 1. The pre-deformation temperature was $250{ }^{\circ} \mathrm{C}$ and the strain rate was $\dot{\varepsilon}=3 \times 10^{-3} \mathrm{~s}^{-1}$.

Conventional metallographic preparation is given in Ref. [8]. Fig. 1 shows the microstructures of samples A-E. The recrystallised volume fraction, $\mathrm{f}_{\text {rec }}$, of samples A-D was measured by the point counting method, Table 1 . The average recrystallised grain size, $d_{\text {rec }}$, and that corresponding to the remaining or non-recrystallised grains, $d_{\text {rem }}$, is given in Table 1 . As it is shown $d_{\text {rec }}$, is similar for samples B to D because $d_{\text {rec }}$ is mainly a function of the ZenerHollomon parameter, i.e., it is independent on the amount of the pre-deformation.

Sample D exhibit high $\mathrm{f}_{\text {rec }}$, but it is difficult to obtain a complete recrystallised microstructure by this procedure. For deformations larger than 0.6 approximately some localization of the deformation (and therefore localization of the recrystallisation) occurs on certain parts of the sample leading to an undesirable heterogeneous grain size distribution along 
the gauge length of the tensile sample. Additionally, tensile deformations larger than 0.6

produce excessive thinning of the sample making difficult their manipulation. Therefore, an additional type of samples, E, was produced by means of 2 rolling passes at $375^{\circ} \mathrm{C}$ of $10 \%$ and $57 \%$ to a final thickness of $1 \mathrm{~mm}$ plus an annealing treatment at $250^{\circ} \mathrm{C} / 30 \mathrm{~min}$. In this case the microstructure appears to be fully recrystallised, Fig. 1e. For the purpose of the present paper, this sample can be considered as the ideal case of "uniform fine grained" microstructure in contrast to sample A which can be considered as "uniform coarse grained" microstructure.
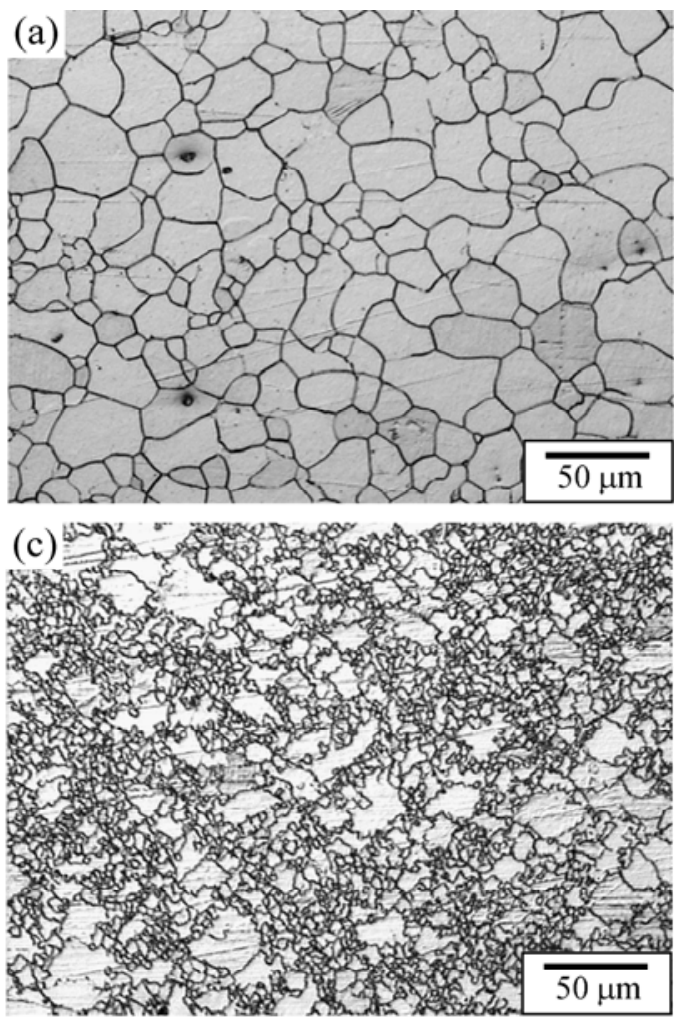
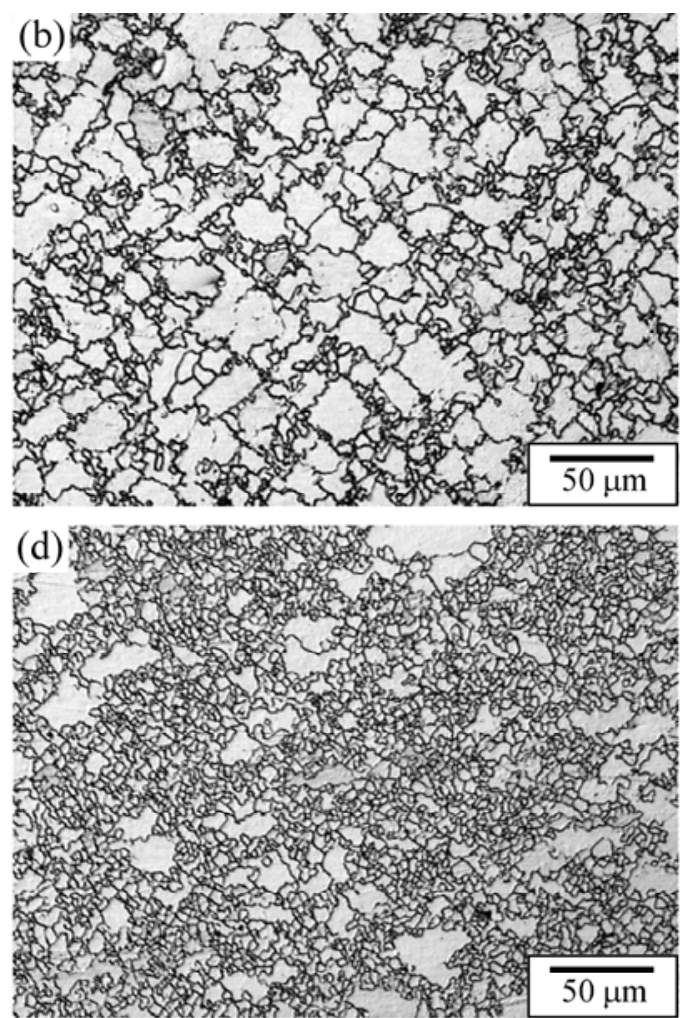

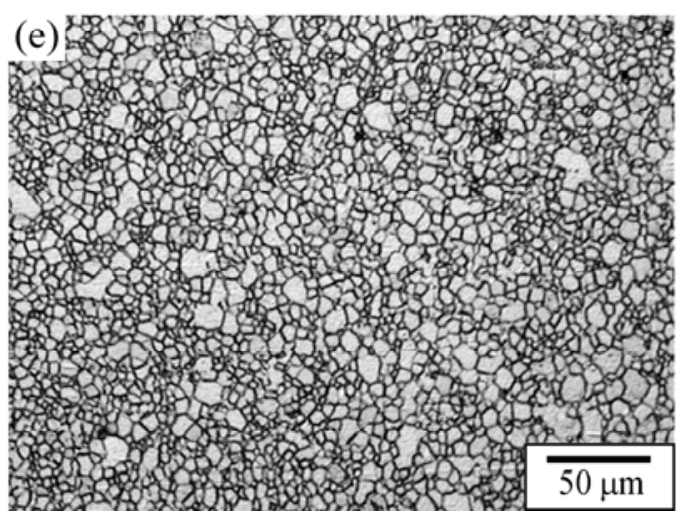

Figure 1: (a)-(e) Micrographs corresponding to samples A-E. 
Strain rate change (SRCH) tests were performed at $250^{\circ} \mathrm{C}$ in samples A-E. In Fig. 2, tests for two D samples are shown A detailed description of these tests was given elsewhere $[8,9]$. Ideally, this kind of tests allows determination of steady state stresses with minor changes in the microstructure. However, some grain growth occurs during deformation at the lower strain rate due to the relatively fine initial recrystallized grain size of $5 \mu \mathrm{m}$. The grain size in the recrystallized zones was measured, points A and B of Fig. 2, by interruption of the test and quenching. The major part of the grain growth occurs before point A. For the later modelization of the creep behavior, grain sizes after the SRCH test (point B of Fig. 2), $d_{a t}$, given in Table 1, may be used as a good approximation of the representative grain size for the regions that have undergone recrystallisation.

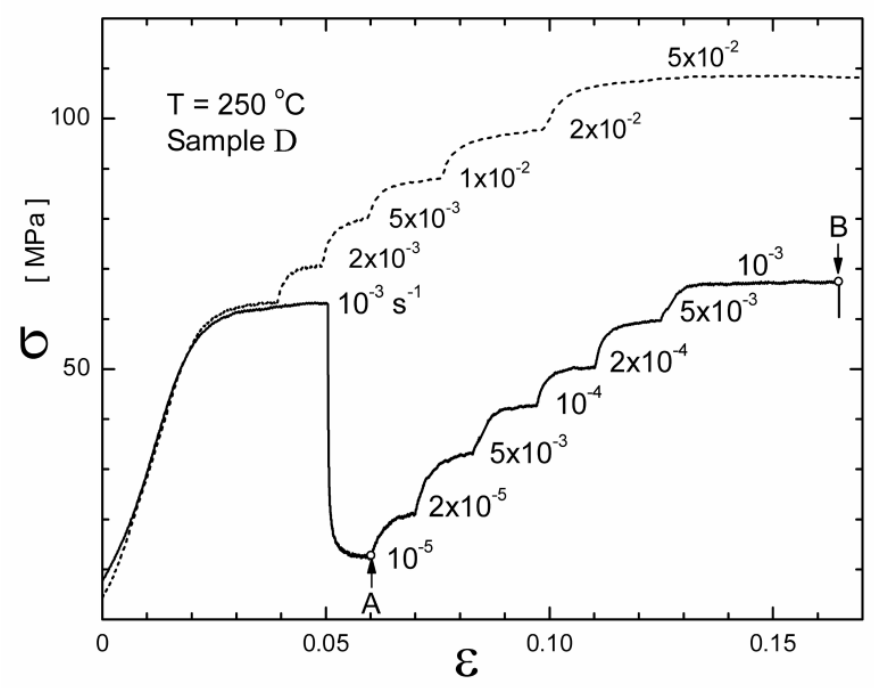

Figure 2: Strain-rate-change test of two D samples. The grain size in the recrystallised zones was measured in points A and B by interruption of the test and quenching. 

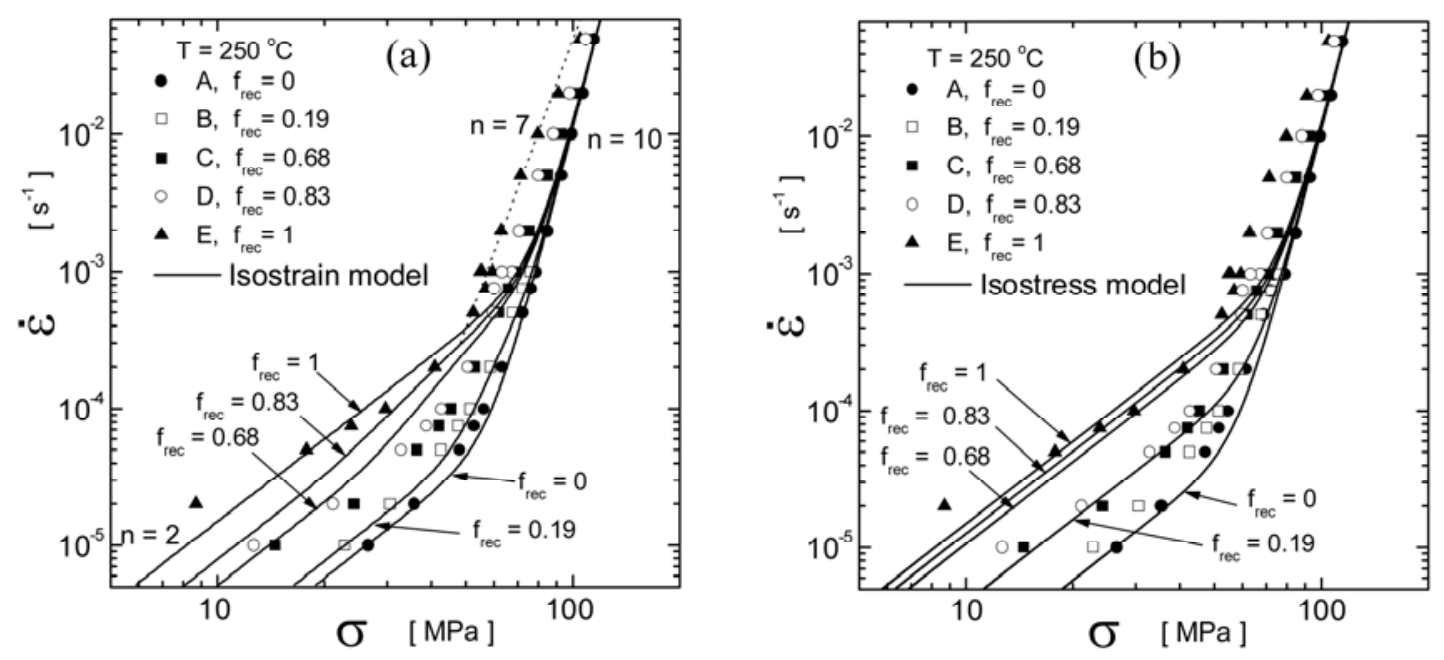

Figure 3: The strain rate against the stress of samples A-E at $250{ }^{\circ} \mathrm{C}$, measured by SRCH tests and curves calculated with (a) the isostrain model and (b) the isostress model.

\section{Results and Discussion}

Figure 3a-b shows strain rate-stress data of samples A-E measured by SRCH tests at $250^{\circ} \mathrm{C}$. The creep behavior is described by the following power law relation between the strain rate and the stress $[10,11]$ :

$$
\dot{\varepsilon}=\operatorname{AD}\left(\frac{\mathrm{b}}{\mathrm{d}}\right)^{\mathrm{P}}\left(\frac{\sigma}{\mathrm{E}}\right)^{\mathrm{n}}
$$

where $\mathrm{A}$ is a material constant, $\mathrm{E}$ is the Young's modulus, $\mathrm{D}=\mathrm{D}_{0} \exp (-\mathrm{Q} / \mathrm{RT})$ is the appropriate diffusion coefficient, $\mathrm{Q}$ is the activation energy, $\mathrm{R}$ is the gas constant, $\mathrm{T}$ is the absolute temperature, $b$ is the Burgers vector, $d$ is the grain size, $n$ is the stress exponent, and $p$ is the grain size exponent.

In Fig. 3, two stress exponent regimes can be distinguished. In the low stress exponent regime, $\mathrm{n}$ is close to two, which corresponds to the GBS mechanism. In the high stress exponent regime, the $\mathrm{n}$ varies between 7 and 10 [8]. Noticeable dependence on the degree of recrystallization is evident in both regimes. Variations of the flow stresses with the grain size in the high exponent range are not expected. This could be attributed to an effect of the initial grain size on the dynamic recrystallisation leading to a decrease in the maximum stress in the alloy with the finest grain size [12]. In the present work the analysis is focused on the 
low exponent regime. In the following, the predictions of two deformation models, based on the isostrain and the isostress conditions, are compared with these experimental data.

Ghosh and Raj [6] proposed a model to considering that each grain, of size $d_{i}$, deforms at the imposed macroscopic strain rate, $\varepsilon_{\mathrm{i}}=\dot{\varepsilon}$; i.e., an isostrain condition is assumed. The stress supported by these grains, $\sigma_{\mathrm{i}}$, can be related to the strain rate according to:

$$
\dot{\varepsilon}=k_{1}\left(\frac{b}{d_{i}}\right)^{2}\left(\frac{\sigma_{i}}{E}\right)^{2}+k_{2}\left(\frac{\sigma_{i}}{E}\right)^{n}
$$

where the added creep laws correspond to GBS and slip-creep having parameters to be determined in both the low and high stress exponent regimes. The stress supported by these grains, $\sigma_{\mathrm{i}}$, contributes to the overall stress $\sigma$ weighted with their volume fraction:

$$
\sigma=\sum_{i} f_{i} \sigma_{i}
$$

where $f_{i}$ denotes the volume fraction of grains having grain size $d_{i}$.

The procedure to obtain a curve $(\dot{\varepsilon}, \sigma)$ is as follows: For a given input value of $\dot{\varepsilon}$, the stress $\sigma_{\mathrm{i}}$ is computed for each grain size $\mathrm{d}_{\mathrm{i}}$, using Eq.(4). The total flow stress $\sigma$ is next calculated with Eq. (5) using the respective volume fractions.

On the other hand, Ponge and Gottstein [7] modelize the softening during dynamic recrystallisation taking into account the contribution of GBS from the new fine recrystallised grains. According to these authors, the isostrain approach is the correct one as long as the necklace structure of the recrystallised grains is not extended contiguously throughout the cross section of the specimen. Ponge and Gottstein proposed that for values of $f_{\text {rec }}$ larger than 0.1 the percolation of the necklace structure occurs and the constituents can deform independently; i.e., an isostress condition is assumed.

This isostress model assumes that the stress supported by the grains of class $i, \sigma_{i}$, is equal to the applied stress $\sigma$. The deformation of these grains is given by

$$
\dot{\varepsilon}_{\mathrm{i}}=\mathrm{k}_{1}\left(\frac{\mathrm{b}}{\mathrm{d}_{\mathrm{i}}}\right)^{2}\left(\frac{\sigma}{\mathrm{E}}\right)^{2}+\mathrm{k}_{2}\left(\frac{\sigma}{\mathrm{E}}\right)^{\mathrm{n}}
$$


The overall deformation is given by:

$\dot{\varepsilon}=\sum_{\mathrm{i}} \mathrm{f}_{\mathrm{i}} \dot{\varepsilon}_{\mathrm{i}}$

The procedure to obtain a curve $(\dot{\varepsilon}, \sigma)$ is straightforward in this case: for a given input value of $\sigma$, the strain rate $\dot{\varepsilon}_{\mathrm{i}}$ is computed for each grain size $\mathrm{d}_{\mathrm{i}}$, using Eq.(6). The total strain rate $\dot{\varepsilon}$ is calculated with Eq.(7).

The fitting of Eq.(1) using data from samples A and E with quite homogeneous grain sizes shows that an exponent $\mathrm{p}=2$ is suitable for the AZ31 alloy. Furthermore, the parameters $\mathrm{k}_{1}$ and $\mathrm{k}_{2}$ were determined by fitting the data of Fig. 3a, for sample A. These parameters are $\mathrm{k}_{1}$ $=1.3 \times 10^{11} \mathrm{~s}^{-1}$ and $\mathrm{k}_{2}=3 \times 10^{24} \mathrm{~s}^{-1}$, a exponent $\mathrm{n}=10$ was used in the high stress exponent regime.

With the values of $k_{1}$ and $k_{2}$ fixed, a set of curves is obtained as continuous lines in Fig. 3a and $b$ for both models. For simplicity, the microstructure was modeled as composed of two classes of grains, non-recrystallised and recrystallised, with average grain sizes of $d_{1}=25.7 \mu \mathrm{m}$ and $\mathrm{d}_{2}=8 \mu \mathrm{m}$, respectively, and the volume fractions given in Table 1 . The grain size of $8 \mu \mathrm{m}$ was chosen as representative of the fine grains because is close to $d_{a t}$, and also gives a reasonable fit of sample E data in Fig. 3.

The upper and lower predicted lines of Figs 3a and 3b corresponds to the idealized cases of "fine grained" and "coarse grained" microstructures, respectively. As the volume fraction of fine grains, $\mathrm{f}_{\text {rec }}$, increases the volume fraction of coarse grains $\left(=1-\mathrm{f}_{\text {rec }}\right)$ decreases. This moves the curves of Fig. 3 gradually toward higher strain rates. From the comparison of Fig. 3a against Fig. 3b it can be concluded that both models predict a quite different behavior during the transition from coarse, $d=25.7 \mu \mathrm{m}$, to fine grains, $\mathrm{d}=8 \mu \mathrm{m}$, as the volume fraction of fine grains increases. In the isostrain model, a relatively low volume fraction of fine grains $\left(\mathrm{f}_{\text {rec }}=\right.$ 0.19) produces a small increase in strain rate, while in the isostress model the predicted effect of this contribution is much more pronounced. The contrary occurs with the contribution of coarse grains: a low fraction of coarse grains produces a noticeable decrease in strain rate in the isostrain model while a small decrease is predicted in the isostress model. As can be seen in Fig. 
3 the overall data behavior shows that the contribution to the strain rate of fine grains increases slowly as predicted by the isostrain model. This is clearly seen in the slight increase of strain rate, from samples A to B in Fig. 3a.

To fulfill the conditions of the isostress model (phases deforming independently) the percolation of the softer phase is required [7]. A percolation threshold of $\mathrm{f}_{\text {rec }} \approx 0.1$, was originally proposed [7]. Therefore, an isostress behavior is expected for almost all the samples investigated B, C and D. However, it can be seen that for $f_{\text {rec }}=0.19$ (samples B) the recrystallised grains have not reached the percolation situation with the finer grains covering uniformly the coarse grains and forming percolation paths. Considering the microstructure aspect, it could be expected an isostress conditions on samples D, perhaps the coexistence of models for sample C and a isostrain condition for sample B. In the case of D samples, the strain rates are lower than predicted with both models. It should be pointed out that there is a possible additional effect due to the form of the recrystallised grains. As can be seen in Fig. 1, the recrystallized grains have an irregular form while grains in sample $\mathrm{E}$ are almost spherical. Taking into account the importance of the accommodation phenomena in the GBS mechanism, this irregular form of the grains could represent an impediment for GBS in samples $\mathrm{C}$ and $\mathrm{D}$ with respect to the ideal behavior considered in the isostrain or isostress models and leading to lower than predicted strain rates

\section{Conclusions}

Summarizing, the influence of a bimodal grain size distribution on creep behavior during the superplastic regime has been analyzed, for the magnesium alloy AZ31, by means of the isostrain model of Ghosh and Raj [6] modified by del Valle and Ruano [8] and the isostress model of Ponge and Gottstein [7]. The isostrain model satisfactorily fits the experimental data for low-medium recrystallised volume fractions. This result allows further rationalization of the creep behavior of materials with bimodal grain size distribution. 


\section{Acknowledgments}

The authors acknowledge financial support from CICYT under program MAT2006-02672 and from the Commanded Autónoma de Madrid under program 2006/60M067. JAV acknowledges support from a Ramón y Cajal contract awarded by the Spanish Ministry of Education and Science.

\section{References}

[1] A. Galiyev, R. Kaibyshev, Scripta Mater., 51 (2004) 89-93.

[2] R. Lapovok, P.F. Thomson, R. Cottam, Y. Estrin, Mater. Sci. Eng. A 410-411 ( 2005) 390-393.

[3] R.B. Figueiredo, T.G. Langdon, Mater. Sci. Eng. A 430 (2006) 151-156.

[4] J.J. Blandin, R. Dendievel, Acta Mater. 48 (2000) 1541-1549.

[5] J.C. Tan, M.J. Tan, Mater. Sci. Eng. A 339 (2003) 124-132.

[6] A.K. Ghosh, R. Raj, Acta Metall. 29 (1981) 607-616.

[7] D. Ponge, G. Gottstein, Acta Mater. 46 (1998) 69-80.

[8] J.A. del Valle, O.A. Ruano, Acta Mater. 55 (2007) 455-466.

[9] J.A. del Valle, M.T. Perez-Prado, O.A. Ruano. Metall. Mater. Trans. A, 36 (2005) 1427-1438.

[10] W.J. Kim, S.W. Chung, C.S. Chung, D. Kum, Acta Mater. 49 (2001) 3337-3345.

[11] O.A. Ruano, A.K. Miller, O.D. Sherby, Mater. Sci. Eng. 51 (1981) 9-16.

[12] M.R. Barnett, A.G. Beer, D. Atwell, A.Oudin. Scripta Mater. 51 (2004) 19-24. 